\title{
छூ \\ Tip-Enhanced Raman Spectroscopy and Microscopy on Single Dye Molecules with 15 nm Resolution
}

\author{
Jens Steidtner and Bruno Pettinger* \\ Fritz-Haber-Institut der Max-Planck-Gesellschaft, Faradayweg 4-6, D-14195 Berlin, Germany \\ (Received 15 February 2008; revised manuscript received 17 April 2008; published 9 June 2008)
}

\begin{abstract}
Our recently developed approach of UHV-tip-enhanced Raman spectroscopy permits us to acquire Raman spectra of a few single brilliant cresyl blue (BCB) molecules and even a single one adsorbed on a $\mathrm{Au}(111)$ surface. This is substantiated by simultaneously recorded STM images. Furthermore, because of the reduced photobleaching in UHV, the time frame for spectral acquisition is sufficiently extended to allow tip-enhanced Raman imaging of a single BCB molecule with a lateral resolution of $15 \mathrm{~nm}$.
\end{abstract}

PACS numbers: 68.37.Uv, 33.20.Fb, 68.43.Pq, 73.20.Mf

With the invention of scanning tunneling [1] and atomic force microscopy [2] the characterization of surfaces with atomic resolution has become feasible. In the form of inelastic electron tunneling spectroscopy (IETS) the former technique permits chemical identification of molecules and even single molecules [3-5]; this can, however, only be achieved using cryogenic temperatures; moreover, at present IET spectra have a rather poor fingerprint quality. Optical spectroscopies, on the other, hand can provide detailed and rich chemical information about an adsorbate in a wide temperature range. However, for studies in the nanometer region one has to overcome limitations of the low sensitivity of standard Raman spectroscopy and the diffraction limited resolution of light. Both of these restrictions can be surmounted by generating and using enhanced, narrow-sized electromagnetic near fields in the vicinity of a metallic tip, which enable both ultrahigh sensitivity and spatial resolution to be achieved. In the first papers on tip-enhanced Raman spectroscopy (TERS) [68], enhancement factors of about $\sim 10^{4}$ were reported. More recently, enhancement factors of $10^{6}-10^{9}$ have been observed [9-11]. With such high sensitivities the spectroscopical detection of individual molecules seems feasible. Indeed, there are recent reports on singlemolecule TERS [10-13], based, however, only on indirect evidence such as spectral fluctuations.

For 1D objects such as carbon nanotubes, Anderson et al. demonstrate enhanced Raman spectroscopy and mi-

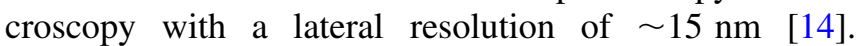
Theoretical calculations indicate a lateral resolution of $\sim 8 \mathrm{~nm}$ for a gold tip of $20 \mathrm{~nm}$ radius above a gold substrate [15]; other authors even consider molecular resolution possible [16].

In this Letter we present a tip-enhanced Raman spectroscopic and microscopic study on brilliant cresyl blue (BCB) adsorbed on gold single-crystal surfaces performed in ultrahigh vacuum (UHV). Because of substantially reduced photobleaching under UHV conditions, we are able not only to record TER spectra of single dye molecules, but also Raman images of a single dye molecule with a resolution of a few nm.
The UHV-TERS and TERS imaging system employed here has been described recently [17]. It mainly consists of a DME Polyscope 500 UHV-STM and an optical setup employing a parabolic mirror with a numerical aperture of $\mathrm{NA}=1$ and a surface quality of $\lambda / 4$ made by Rodenstock Präzisionsoptik, Germany, for focusing the laser and collecting the Raman light. The laser source is a HeNe-laser with a wavelength of $633 \mathrm{~nm}$ and a linear polarization mode which is converted into a pseudoradial polarization mode by an arrangement of four $\lambda / 2$ retarders [18]. Radial polarization combined with a high numerical aperture parabolic mirror leads to a sharp focus $(\sim \lambda / 2)[19,20]$ as well as to an increased longitudinal electric field component along the tip axis [21,22]; such focal properties are essential for a high field enhancement $[23,24]$.

For spectral analysis a Spex Triplemate spectrograph equipped with a Princeton Instruments Spec-10 liquid nitrogen cooled charge coupled device was used. Spectra were acquired with a laser power of $0.4 \mathrm{~mW}$ and an integration time of $1 \mathrm{~s}$ and images with $0.5 \mathrm{~s}$ per data pixel. Tips were etched from a gold wire of $0.25 \mathrm{~mm}$ diameter in a solution of $1: 1$ ethanol/fuming $\mathrm{HCl}$ [25]. This yields smooth tips with radii of $15-20 \mathrm{~nm}$ as indicated by scanning electron microscopy images (not shown). After each experiment the tip was checked for cleanliness. The bias was $+150 \mathrm{mV}$ and the tunnel current was set to $1 \mathrm{nA}$. Au single crystals were obtained from Mateck. They were cleaned in piranha solution and annealed by inductive heating in an Ar atmosphere. BCB (Fluka) was adsorbed from a $10^{-6} \mathrm{M}$ or, for lower coverages, $10^{-8} \mathrm{M}$ solution in Millipore water. The crystals were subsequently rinsed with pure Millipore water to remove excess BCB molecules. The UHV experiments were performed at pressures in the $10^{-10}$ mbar range. For measurements in an oxygen atmosphere the chamber was vented with oxygen 5.5.

With this experimental setup a focal spot with a diameter of $300 \mathrm{~nm}$ is achieved [17]. The tip radius of $15 \mathrm{~nm}$ leads to a diameter of the TERS region of $15 \mathrm{~nm}$ [26].

$\mathrm{BCB}$ is an organic dye that has electronic transitions near to the resonance of the localized surface plasmon modes of the tunneling gap between the gold tip and 
gold sample, making it an ideal model system. Its resonant excitation with a $633 \mathrm{~nm}$ wavelength laser produces a weak, but detectable resonance Raman signal (far-field), while its adsorption at a metal surface effectively quenches the dye fluorescence. Because of our preparation procedure, on atomically flat Au surfaces about a 1/2 monolayer of BCB is adsorbed, corresponding to about 36000 molecules in the focal area [27]. Upon tip approach, the signal intensity of the strongest band at $570 \mathrm{~cm}^{-1}$ raises to a 3900 -fold level corresponding to a Raman enhancement of $1.6 \times 10^{6}$. The corresponding spectra show strong intensities though the TER signal stems from only $\sim 90$ molecules located within the TERS region (see Fig. 4 below). In this experiment we are still about 2 orders of magnitude short of the detection of single molecules; this indicates single-molecule TERS is in reach.

In the experiments shown in Figs. 1(a)-1(d), regions of a gold surface covered with either five $\mathrm{BCB}$ molecules or only a single, well separated one, were imaged by scanning tunneling microscopy. The STM height profiles [Figs. 1(b)-1(e)] display BCB molecules with a lateral size of $1.4 \times 0.7 \mathrm{~nm}^{2}$ and an effective height of 120 $140 \mathrm{pm}$. TER spectra were recorded with the molecules located exactly in the gap between gold tip and substrate [Figs. 1(c)-1(f)]. For 90 molecules a Raman intensity of 7800 counts/s is observed at $570 \mathrm{~cm}^{-1}$, which corresponds to an intensity of $\approx 87$ counts/s for one molecule. Thus, the signal intensities of 410 counts/s for five and 110 counts/s for a single molecule are in good agreement with the number of probed molecules. Note that these spectra were recorded with different tips providing a different Raman enhancement factor. After retraction of the tip by $1 \mu \mathrm{m}$ a far-field Raman signal of the investigated surface area could not be detected for the low BCB coverages. Since single-molecule spectra of BCB were recorded with an integration time of only $1 \mathrm{~s}$, the sensitivity also allows Raman imaging of single molecules on a time scale in which the probed surface section is stable.
For the single molecule in Fig. 1(c), Fig. 2(a) displays the local intensity of the band at $570 \mathrm{~cm}^{-1}$ during a scan across the surface section. Under the present experimental conditions, i.e., a well-defined hot spot and a large suppression of diffusion by adsorption on a metal surface, probably on a specific site, there is no spectral blinking that is often observed in surface-enhanced single-molecule Raman spectra. After performing the Raman mapping, a STM image of essentially the same area was recorded, still showing the same single molecule (not shown). Figure 2(b) shows the horizontal and vertical TER intensity cross sections through the center of the molecule as well as a theoretical curve of the Raman enhancement profile along the substrate surface following a simple model. It describes the enhancement as the near-field interaction of an oscillating dipole in the center of a spherical particle with a molecule [28]. The electromagnetic coupling of the tip and the substrate is neglected which could result in an even more confined near field. Any deviation from a perfect shape of the tip cone or apex may result in a contrary effect. Therefore, the simplified model probably yields a realistic distance dependence of TERS as the $r^{-12}$ dependence dominates all other effects [26]. The Raman intensity $I(r)$ is given by

$$
I(r)=I_{0}\left(1+\frac{r^{2}}{\left(R_{s}+d\right)^{2}}\right)^{-6}+I_{b g},
$$

where $r$ is the lateral distance from the molecule to the central point underneath the tip. A tip radius of $R_{s}=$ $20 \mathrm{~nm}$ and a tunneling gap width of $d=1 \mathrm{~nm}$ were assumed. The values for $I_{0}$ and $I_{b g}$ were chosen in correspondence with the spectra of the single $\mathrm{BCB}$ molecule and the tip above the uncovered gold sample in Fig. 1(f). Note that the probe is scanned across a homogeneous surface on which only a single Raman scatterer is placed. Thus, the local signal intensity consists of a constant background caused by the probe itself and the contribution of the scatterer. The latter is determined by the tip-scatterer distance dependent Raman enhancement, which exhibits an
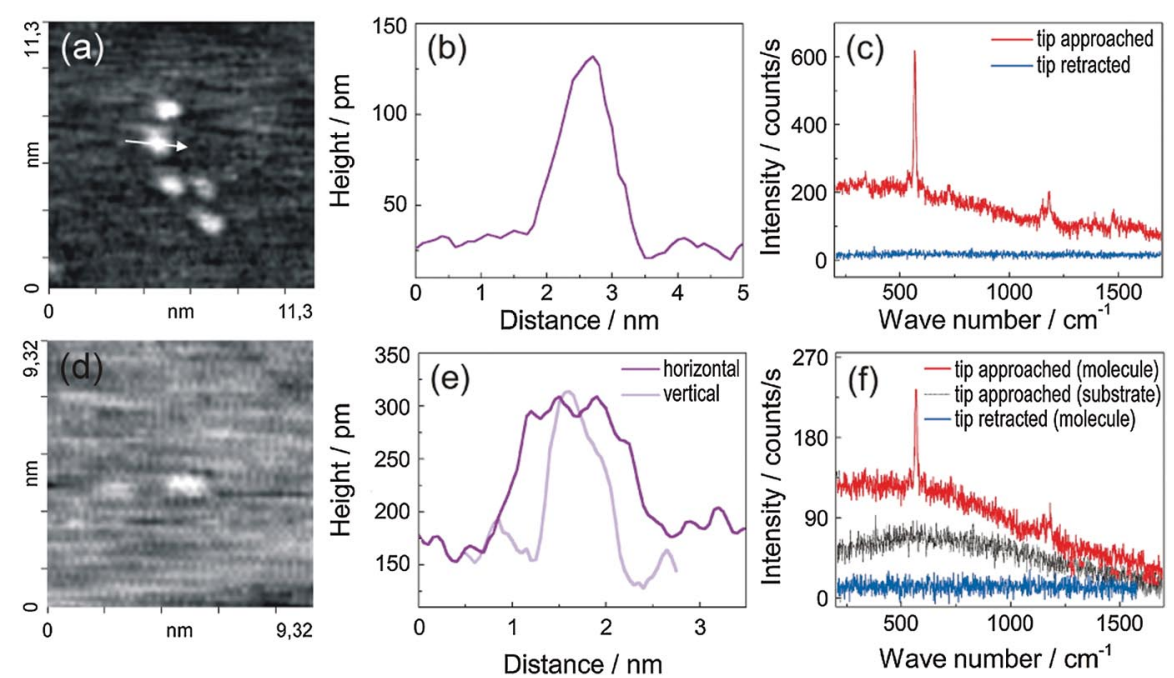

FIG. 1 (color). STM images of five (a) and a single BCB molecule (d) adsorbed on $\mathrm{Au}(111)$, the corresponding height cross sections (b),(e) and the UHV-RR and UHV-TERR spectra (c),(f). 


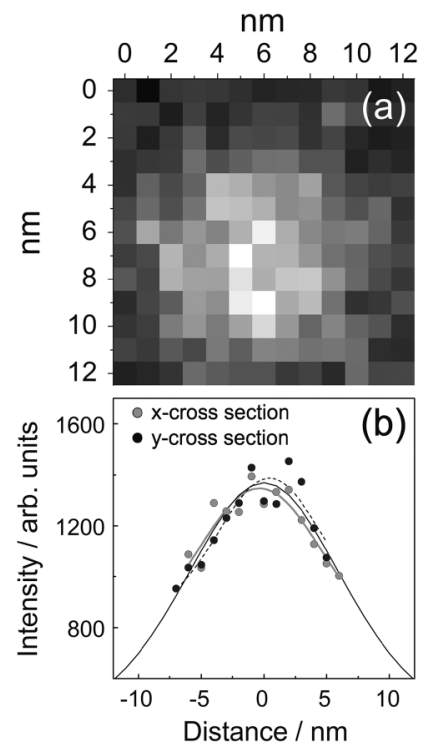

FIG. 2. (a) TERS image in Raman mode $\left(568-572 \mathrm{~cm}^{-1}\right)$ of a single BCB molecule on $\mathrm{Au}(111)$; (b) $\mathrm{x}$ - and $\mathrm{y}$-cross sections of the Raman intensity through the center of the molecule.

$r^{-12}$ dependence [26]. Consequently, the Raman image displays the above TERS profile along the surface. The agreement of the intensity cross sections with the theoretical curve confirms the validity of the simplified model employed here. Based on the same model the resolution of TERS imaging can be estimated by calculation of the Raman intensity map of two equal point scatterers placed on a plane surface [29]. If we assume that the intensity at the minimum along the line between the two scatterers should not exceed the maximum intensity of a single separated scatterer, we can postulate a simple rule for the image resolution of TERS: The minimum distance $\Delta x_{\min }$ that can still be resolved, is approximately $70 \%$ of the sum of tip radius $R_{s}$ and tunneling gap width $d$.

Another example for tip-enhanced Raman mapping is presented in Fig. 3. The STM image shows a $\mathrm{Au}(111)$ surface with a few monoatomic steps at which BCB molecule clusters are preferentially adsorbed. ATER scan of the intensity at $570 \mathrm{~cm}^{-1}$ clearly resolves the position of these clusters that are separated by distances of 15-20 nm.

Above we presented single-molecule Raman imaging, which requires the dye to remain stable during the exposure of the enhanced field; i.e., the photobleaching must be substantially reduced under UHV conditions. However, for resonantly excited dye molecules, the huge electromagnetic enhancement also boosts the rate of photobleaching of dye molecules, with bleaching time constants (in air) down to subseconds $[9,26]$ often yielding deteriorated spectra due to photodegradation of the dye. Under vacuum conditions, Raman studies on dye molecules without nearfield enhancement yield a delayed photobleaching [30]. That this also holds for strongly enhanced near fields, will be shown in the following experiment.

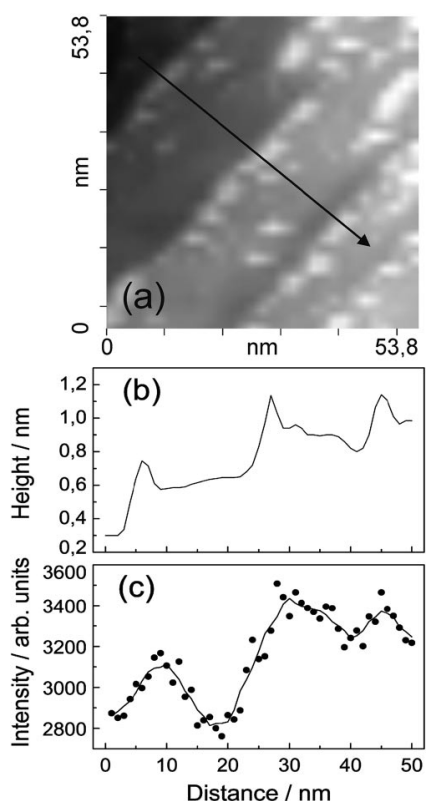

FIG. 3. STM image of BCB molecules adsorbed on step sites of a $\mathrm{Au}(111)$ sample; (b) topography profile along the arrow; (c) Raman intensity $\left(568-572 \mathrm{~cm}^{-1}\right)$ profile along the arrow.

Besides these benefits, the pronounced resonance of the system also causes a rapid photobleaching of the dyes in the vicinity of the tip. To determine the rate of photobleaching under UHV conditions, 30 subsequent spectra of a BCB monolayer on $\mathrm{Au}(111)$ were recorded first. The next experiment was performed with a freshly prepared sample and the same tip, but after filling the chamber with $1 \mathrm{~atm}$ of pure oxygen. Both series of spectra are shown in Figs. 4(a) and 4(b). In Fig. 4(c) the signal and background intensities at about $570 \mathrm{~cm}^{-1}$ are plotted vs the time of illumination showing a very different bleaching behavior in the presence and absence of oxygen. In UHV, the band intensity only drops to $\sim 74 \%$ within $30 \mathrm{~s}$ and all spectral features remain clearly visible. By contrast, the adsorbate is strongly and rapidly bleached in an $\mathrm{O}_{2}$ atmosphere which already affects the first spectrum. Almost the complete spectral information is lost during further illumination of the sample; after $30 \mathrm{~s}$ the band intensity is reduced to $<15 \%$ of the initial value. Figure 4(c) shows an initial time constant of photobleaching in an oxidative atmosphere of only $2.9 \mathrm{~s}$. Another interesting aspect is the different evolution of the background intensity in UHV and in $\mathrm{O}_{2}$. In UHV, both band and background intensity decrease in almost equal measure. In $\mathrm{O}_{2}$, the rapid decrease of the signal intensity is accompanied by a strong, but temporary increase of the background around $250-1000 \mathrm{~cm}^{-1}$. It reaches a maximum value in approximately $3 \mathrm{~s}$ after which it is also bleached. The absence of this rise of the background under UHV conditions indicates that this effect must be associated with decomposition products of $\mathrm{BCB}$ accumulated during rapid bleaching, which are then themselves exposed to a further photodecomposition pro- 


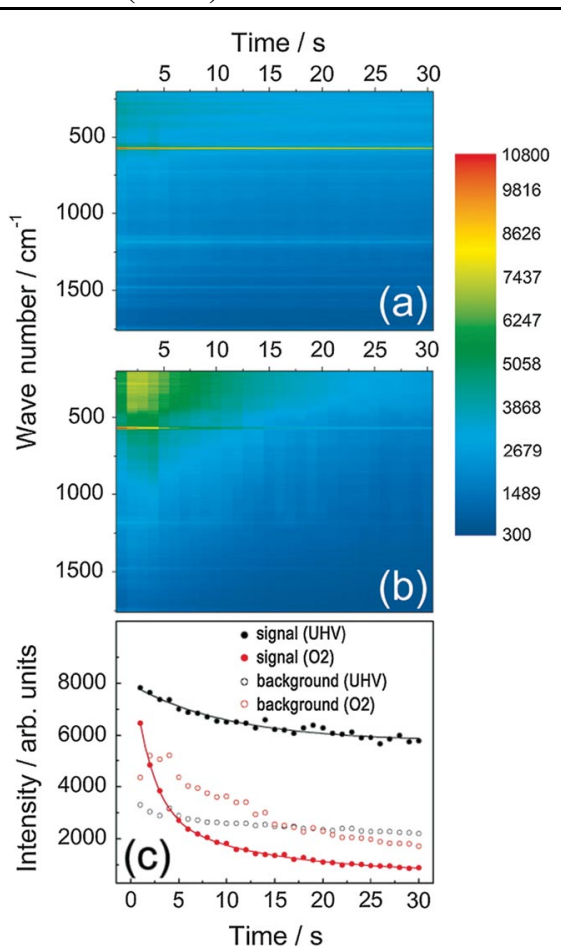

FIG. 4 (color). Series of 30 subsequent TERR spectra of a $\mathrm{BCB}$ monolayer on $\mathrm{Au}(111)$ (integration time: $1 \mathrm{~s}$; laser power: $0.4 \mathrm{~mW}$ ) recorded (a) in ultrahigh vacuum and (b) in a pure oxygen atmosphere; (c) time dependence of the TER band and background intensity at $570 \mathrm{~cm}^{-1}$ in the spectra.

cess. Under vacuum conditions the rate of photobleaching is too low to visibly affect the background intensity.

Because of the extremely sharp focus of the parabolic mirror with high numerical aperture as well as the use of smooth and sharp gold tips, an excellent contrast between the far-field and near-field signal of $\approx 3900$ is achieved with the present UHV-TERS setup, employing monocrystalline Au samples as substrates. The strong contrast indicates an electromagnetic Raman enhancement of $\sim 10^{6}$ which is sufficient for the detection of single resonant dye molecules even within integration times as short as $1 \mathrm{~s}$. The Raman spectra can be assigned to their source via the topographic images of the STM. The Raman mapping yields chemical information with a lateral resolution of $\sim 15 \mathrm{~nm}$ as proven by TERS imaging of a single BCB molecule as well as of larger molecular ensembles. We showed that, under UHV conditions, the adsorbed dye molecules are much more stable against photobleaching than in an oxygen atmosphere, even if very strong nearfield intensities are present. Thus, in UHV a substantially extended measurement time is available, which permits Raman mapping (TER microscopy) on a sample.

The authors thank Professor Gerhard Ertl for interesting and helpful discussions. *pettinger@fhi-berlin.mpg.de

[1] G. Binnig, H. Rohrer, C. Gerber, and E. Weibel, Appl. Phys. Lett. 40, 178 (1982).

[2] G. Binnig, C. F. Quate, and C. Gerber, Phys. Rev. Lett. 56, 930 (1986).

[3] B. C. Stipe, M. A. Rezaei, and W. Ho, Science 280, 1732 (1998).

[4] W. Y. Wang, T. Lee, I. Kretzschmar, and M. A. Reed, Nano Lett. 4, 643 (2004).

[5] T. Komeda, Prog. Surf. Sci. 78, 41 (2005).

[6] R. M. Stöckle, Y.D. Suh, V. Deckert, and R. Zenobi, Chem. Phys. Lett. 318, 131 (2000).

[7] N. Hayazawa, Y. Inouye, Z. Sekhat, and S. Kawata, Opt. Commun. 183, 333 (2000).

[8] B. Pettinger, G. Picardi, R. Schuster, and G. Ertl, Electrochemistry 68, 942 (2000).

[9] B. Pettinger, B. Ren, G. Picardi, R. Schuster, and G. Ertl, Phys. Rev. Lett. 92, 096101 (2004).

[10] K. F. Domke, D. Zhang, and B. Pettinger, J. Am. Chem. Soc. 128, 14721 (2006).

[11] C.C. Neacsu, J. Dreyer, N. Behr, and M.B. Raschke, Phys. Rev. B 73, 193406 (2006).

[12] N. Hayazawa, H. Watanabe, Y. Saito, and S. Kawata, J. Chem. Phys. 125, 244706 (2006).

[13] W. H. Zhang, B. S. Yeo, T. Schmid, and R. Zenobi, J. Phys. Chem. C 111, 1733 (2007).

[14] N. Anderson, A. Hartschuh, S. Cronin, and L. Novotny, J. Am. Chem. Soc. 127, 2533 (2005).

[15] D. Richards, R. G. Milner, F. Huang, and F. Festy, J. Raman Spectrosc. 34, 663 (2003).

[16] A. Downes, D. Salter, and A. Elfick, J. Phys. Chem. B 110, 6692 (2006).

[17] J. Steidtner and B. Pettinger, Rev. Sci. Instrum. 78, 103104 (2007).

[18] R. Dorn, S. Quabis, and G. Leuchs, Phys. Rev. Lett. 91, 233901 (2003).

[19] S. Quabis, R. Dorn, M. Eberler, O. Glckl, and G. Leuchs, Opt. Commun. 179, 1 (2000).

[20] C. Debus, M. A. Lieb, A. Drechsler, and A. J. Meixner, J. Microsc. 210, 203 (2003).

[21] K. S. Youngworth and T. G. Brown, Opt. Express 7, 77 (2000).

[22] M. A. Lieb and A. J. Meixner, Opt. Express 8, 458 (2001).

[23] L. Novotny, R. X. Bian, and X. S. Xie, Phys. Rev. Lett. 79, 645 (1997).

[24] L. Vaccaro, L. Aeschimann, U. Staufer, H. P. Herzig, and R. Dandliker, Appl. Phys. Lett. 83, 584 (2003).

[25] B. Ren, G. Picardi, and B. Pettinger, Rev. Sci. Instrum. 75, 837 (2004).

[26] B. Pettinger, B. Ren, G. Picardi, R. Schuster, and G. Ertl, J. Raman Spectrosc. 36, 541 (2005).

[27] B. Pettinger, G. Picardi, R. Schuster, and G. Ertl, Single Mol. 3, 285 (2002).

[28] M. Kerker and C. G. Blatchford, Phys. Rev. B 26, 4052 (1982).

[29] J. Steidtner, Ph.D. thesis, Freie Universitaet Berlin 2007.

[30] L. A. Deschenes and D. A. Vanden Bout, Chem. Phys. Lett. 365, 387 (2002). 